\title{
In-Plane-Gate GaN Transistors for High-Power RF Applications
}

\author{
Giovanni Santoruvo and Elison Matioli, IEEE member
}

\begin{abstract}
In-plane-gate field effect transistors (IPGFETs) offer an innovative device architecture in which the channel conductivity is modulated by the electric field from the two-dimensional electron gas (2DEG) in the two adjacent in-plane gates, isolated by etched trenches. The planar nature of the gate electrode yields a huge reduction in parasitic gate capacitance, which can lead to much higher frequency. Moreover, the fabrication process for these devices is extremely simple and with inherently self-aligned gates.

Here, we combine for the first time the promising architecture of IPGFETs with the exceptional properties of III-Nitrides, such as large carrier density and breakdown field, to reveal their enormous potential for high-power RF devices. AlGaN/GaN IPGFETs demonstrated large drain current up to $1.4 \mathrm{~A} / \mathrm{mm}$ and transconductance up to $665 \mathrm{mS} / \mathrm{mm}$, which are respectively $9 \mathrm{x}$ and 5x-larger than the best IPGFETs demonstrated in other semiconductors. These devices presented excellent gate control with on-off ratio up to $10^{7}$ along with ultra-low capacitances down to $0.7 \mathrm{aF}$, leading to an estimated $f_{T}$ up to $0.89 \mathrm{THz}$. Extremely large breakdown voltage of $500 \mathrm{~V}$ was observed despite their nanoscale dimensions, with small leakage current below 1 nA up to $300 \mathrm{~V}$. These results reveal that III-Nitride IPGFETs offer a promising pathway for future terahertz devices delivering large output powers.
\end{abstract}

Index Terms-In-plane gate, nanowires, high frequency, gate capacitance, GaN, III-Nitrides, HEMTs, 2DEG, terahertz

\section{INTRODUCTION}

$\mathbf{I}$ PGFETs offer an innovative architecture [1] in which the channel and gates lie on the same plane of the 2DEG. The gate control over electrons in the channel is done by the electric field induced from the adjacent 2DEG gates isolated from the channel by a nanoscale etched trench, which results in a small gate capacitance down to a few aF (as predicted by Ref. [2]) and can lead to much higher frequency operation. Gate electrodes are defined with the same ohmic metalization of the source and drain pads, and do not need any gate dielectrics. Thus, these devices present inherently self-aligned processes requiring only two main fabrication steps. IPGFETs demonstrated in the literature are mainly based on narrow-gap III-V heterostructures such as AlGaAs/GaAs at $300 \mathrm{~K}$ [1, 3-8], GaAs/InGaAs/AlGaAs at $300 \mathrm{~K}$ [9], InGaAs/InP at $4 \mathrm{~K}$ [10] and InAlAs at $300 \mathrm{~K}$ [11]. Although a good gate control has been demonstrated, the relatively small electron density in the 2DEG and the very low critical breakdown field in these IIIVs semiconductors yield small output current and breakdown voltage, and consequently limited output power.

This work was supported by the Swiss National Science Foundation under Grant 200021_169362. The authors are with the Power and Wideband-gap Electronics Research Laboratory (POWERlab), Ecole Polytechnique Federale de Lausanne (EPFL) , CH1015, Lausanne, Switzerland (e-mail: giovanni.santoruvo@epfl.ch; elison.matioli@epfl.ch)

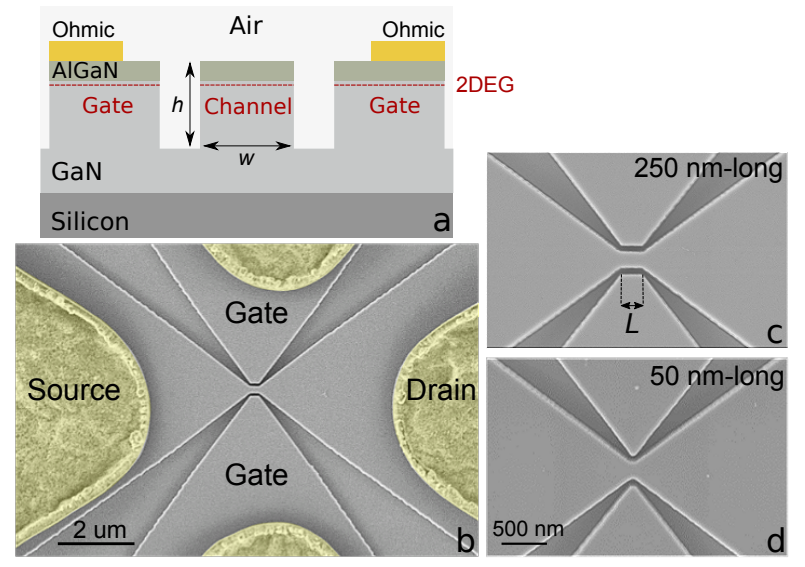

Fig. 1: (a) Schematic and (b) top-view SEM image of a 165 $\mathrm{nm}$-wide and $250 \mathrm{~nm}$-long IPGFET. Zoomed top-view SEM images of a $250 \mathrm{~nm}$-long (c) and $50 \mathrm{~nm}$-long (d) IPGFETs.

In this work, we combine the promising architecture of IPGFETs with the exceptional properties of III-Nitrides to reveal their enormous potential to deliver large output power at very high frequencies. We demonstrate for the first time highperformance IPGFETs on $\mathrm{AlGaN} / \mathrm{GaN}$ heterostructures on silicon substrates with very large $I_{d s}$ of $1.4 \mathrm{~A} / \mathrm{mm}$ and $g_{m}$ up to $665 \mathrm{mS} / \mathrm{mm}$, which are respectively $9 \mathrm{x}-$ and $5 \mathrm{x}$-larger than the best IPGFET demonstrated in other semiconductors [9], and much superior than preliminary works published on $\mathrm{GaN}$ semiconductors [12, 13]. In addition, our devices presented ultra-low gate capacitance down to $0.7 \mathrm{aF}$, high on-off ratio of $10^{7}$, and very high breakdown voltage of $500 \mathrm{~V}$. These results reveal the enormous potential of III-Nitride IPGFETs to deliver large output power in high frequency applications.

\section{RESULTS AND DISCUSSION}

IPGFETs were fabricated on $\mathrm{Al}_{0.25} \mathrm{Ga}_{0.75} \mathrm{~N}(23.5 \mathrm{~nm}) / \mathrm{AlN}$ $(8 \mathrm{~nm}) / \mathrm{GaN}(300 \mathrm{~nm})$ followed by a $3.75 \mu$ m-thick buffer on silicon substrate. The electron mobility $\left(\mu_{e}\right)$, carrier concentration $\left(n_{s}\right)$ and sheet resistance $\left(R_{\mathrm{sh}}\right)$ of the epitaxial structure measured at $300 \mathrm{~K}$ were $1.05 \times 10^{13} \mathrm{~cm}^{-2}, 1690 \mathrm{~cm}^{2} / \mathrm{Vs}$ and 350 $\Omega / \square$, respectively. Their simple fabrication process consists of only two major steps which inherently result in self-aligned gates. The first step is the definition of the channel, gates, source and drain by a single electron beam lithography using hydrogen silsesquioxane $2 \%$ (HSQ), followed by $\mathrm{Cl}_{2}$-based inductively coupled plasma (ICP) etching to isolate the gate and to define mesa regions. We fabricated devices with channel lengths $(L)$ of $250 \mathrm{~nm}$ and $50 \mathrm{~nm}$ and widths $(w)$ varying from

(C) 2017 IEEE. Personal use of this material is permitted. Permission from IEEE must be obtained for all other uses, in any current or future media, including reprinting/republishing this material for advertising or promotional purposes, creating new collective works, for resale or redistribution to servers or lists, or reuse of any copyrighted component of this work in other works. 
$20 \mathrm{~nm}$ to $165 \mathrm{~nm}$. $60 \mathrm{~nm}$-wide isolation trenches were etched with two nominal trench depths $(h)$ of $140 \mathrm{~nm}$ (Shallow) and $210 \mathrm{~nm}$ (Deep) as measured in the mesa regions (the depth within the narrow trenches may be smaller than these values).

The second step is the deposition of ohmic contacts, defined by optical lithography, for source, drain as well as gate

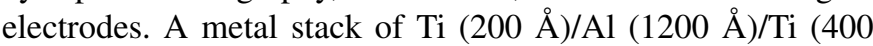
$\AA) \mathrm{Ni}(600 \AA) / \mathrm{Au}(500 \AA)$ was deposited in the contact regions by electron-beam evaporation and annealed at $860^{\circ} \mathrm{C}$. In this work, we focused on demonstrating the simplest fabrication process, without any oxides or passivation layers. Passivations and surface treatments are the subject of our future work. Figures $1 \mathrm{a}$ and $\mathrm{b}$ show the cross-sectional schematic and the top-view SEM image of the device after fabrication. Figures 1c and d show the zoomed SEM view of two different geometries investigated with channel lengths of $250 \mathrm{~nm}$ and $50 \mathrm{~nm}$, respectively.

Figures $2 \mathrm{a}$ and $\mathrm{b}$ show the transfer characteristics for $L$ of $250 \mathrm{~nm}$ and $50 \mathrm{~nm}$, both for the Shallow and Deep samples. For a fixed $L$ and $h$, the $I_{d s}$ and $\left|V_{\text {th }}\right|$ increased for wider channel devices, due to the smaller resistance in wider channels. In addition, the more pronounced strain relaxation of the $\mathrm{AlGaN}$ barrier in narrower channels causes a decrease of carriers induced by piezo-electric polarization fields [14, 15], thus reducing $n_{s}, I_{d s}$ and $\left|V_{\text {th }}\right|$. These devices presented a large on-off ratio up to $10^{7}$ with a small leakage current of less than $10 \mathrm{pA}$ for the Deep and $100 \mathrm{pA}$ for the Shallow devices, revealing an excellent channel control from the inplane gate with air dielectric. For a fixed $w$, an increase in $I_{d s}$ along with a more negative $V_{\text {th }}$ was observed by reducing the length of the nanowires from $250 \mathrm{~nm}$ to $50 \mathrm{~nm}$ (Fig. $2 \mathrm{~b}$ ), which is again expected due to the smaller resistance of the wire and the reduced strain relaxation in the $50 \mathrm{~nm}$-long devices. Double-sweep transfer characteristic measurements (not shown) revealed a $\Delta V_{\text {th }}$ of about $0.2 \mathrm{~V}-0.3 \mathrm{~V}$, which was independent from device geometry and channel sizes. Such $\Delta V_{\text {th }}$ can be associated to the presence of traps in the AlGaN barrier and on the etched sidewalls, which can be mitigated by passivating the device.

The depth of the etched trenches that isolate the gate and channel regions has an important impact on the device performance. By reducing $h$ while maintaining $w$ constant, we observed a significant positive shift in $V_{\text {th }}$ for all device widths, which reveals an increase in gate capacitance for the shallower trenches (discussed in more details later).

Figure $3 \mathrm{a}$ shows the output characteristics of the $50 \mathrm{~nm}$ long $85 \mathrm{~nm}$-wide shallow IPGFET. A large current density $\left(I_{d s} / w\right)$ of $1.4 \mathrm{~A} / \mathrm{mm}$ was observed, which is over $9 \mathrm{x}$-larger than the best IPGFET [9] based on an InGaAs quantum well, due to the much larger carrier density and very small sidewall depletion in III-Nitrides $[16,17]$. Figure $3 b$ shows the transconductance of the $50 \mathrm{~nm}$-long IPGFETs, revealing larger and broader $g_{m}$ for wider nanowires. A significant increase in the absolute value of $g_{m}$ was observed for the Shallow devices, along with a reduction on the width of the $g_{m}$ curves. The normalized $g_{m} / w$ was larger for smaller $w$ due to the improved modulation over the narrower channels, going from $335 \mathrm{mS} / \mathrm{mm}$ for $85 \mathrm{~nm}$-wide channel to $665 \mathrm{mS} / \mathrm{mm}$ for the

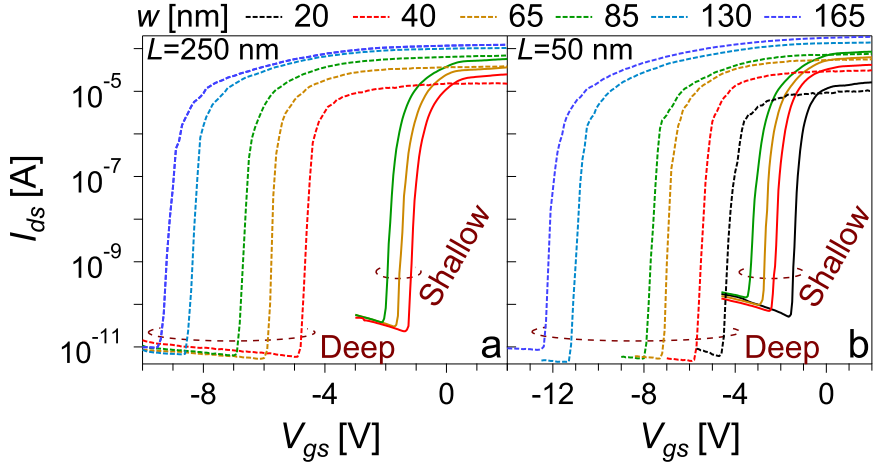

Fig. 2: Transfer characteristics of $250 \mathrm{~nm}-10 n g$ and $50 \mathrm{~nm}-$ long IPGFETs width different widths for Deep (dashed) and Shallow (solid) IPGFETs. Both Deep and Shallow $20 \mathrm{~nm}$-wide 250nm-long IPGFETs were fully depleted.
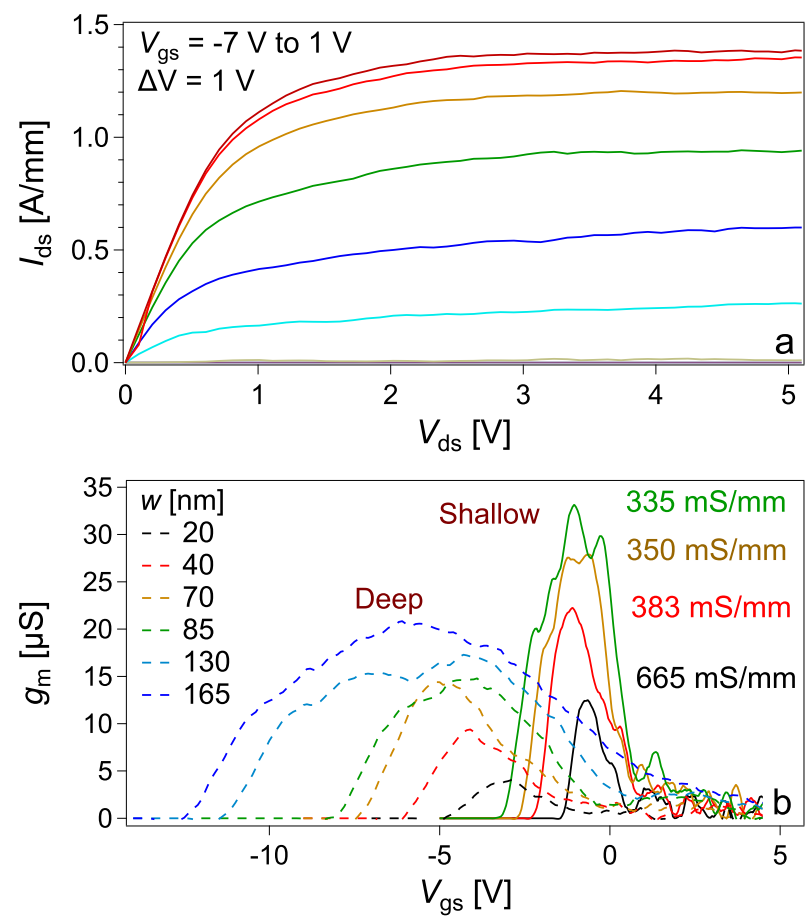

Fig. 3: (a) Output characteristic of a $50 \mathrm{~nm}$-long and $85 \mathrm{~nm}$ wide IPGFET. (b) Transconductance of Deep (dashed) and Shallow (solid) $50 \mathrm{~nm}$-long IPGFETs. The normalized peak transconductance $\left(g_{m} / w\right)$ is shown for the Shallow devices.

$20 \mathrm{~nm}$-wide channel, which is nearly 5x-larger than the best IPGFET [9].

High frequency operations require a small gate capacitance in addition to a large $g_{m}$, which is an intrinsic advantage of IPGFETs. The effective gate-to-channel capacitance per unit of area $\left(C_{\text {eff }}^{\prime \prime}\right)$ was experimentally determined from $n_{s}=1 / q \int_{V_{\mathrm{th}}}^{0} C^{\prime \prime}(v) d v=C_{\mathrm{eff}}^{\prime \prime} \times V_{\mathrm{th}} / q$. We measured $n_{s}$ from Hall bars with similar $w$, and extracted $V_{\text {th }}$ from Fig. 2 at $I_{d s}=$ $1 \mathrm{nA}$. Figure 4a shows the effective gate capacitance per unit of length $C_{\mathrm{eff}}^{\prime}=C_{\mathrm{eff}}^{\prime \prime} \times w$ for the $250 \mathrm{~nm}$-long IPGFETs, which includes contributions from intrinsic and extrinsic capacitances. The intrinsic $C_{\text {int }}^{\prime}$ was numerically calculated using COMSOL (blue curve in Fig. 4a), considering the device cross-section 


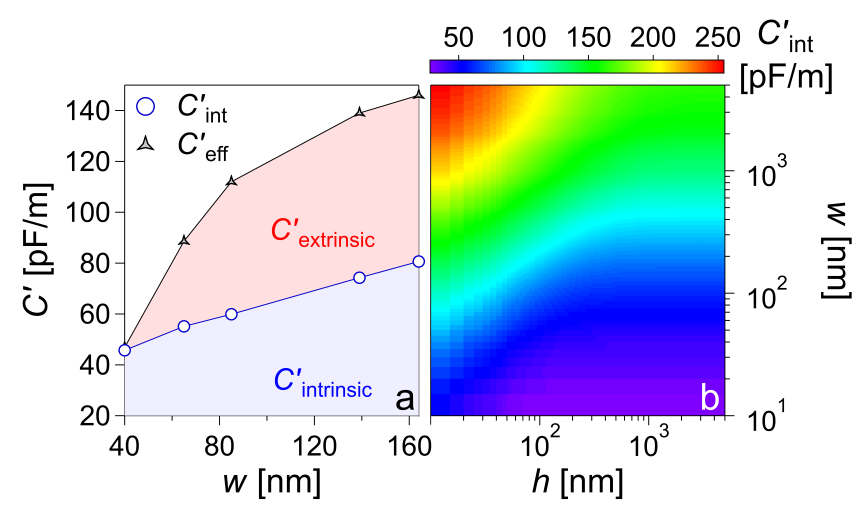

Fig. 4: (a) Measured $C_{\text {eff }}^{\prime}$ (black) and simulated $C_{\text {int }}^{\prime}$ (blue) per unit of length versus $w$ for the $250 \mathrm{~nm}$-long Deep IPGFET. (b) Simulated $C_{\text {int }}^{\prime}$ versus $w$ and $h$.

TABLE I: Characteristics of the $50 \mathrm{~nm}$-long Shallow and Deep IPGFETs. $f_{T}$ was estimated as $f_{T} \approx g_{m} / 2 \pi C_{\text {eff. }}$.

\begin{tabular}{|c|c|c|c|c|c|}
\hline & $w[\mathrm{~nm}]$ & $n_{s}\left[e 12 \mathrm{~cm}^{-2}\right]$ & $C_{\text {eff }}[\mathrm{aF}]$ & $g_{m}[\mu \mathrm{S}]$ & $f_{T}[\mathrm{THz}]$ \\
\hline \multirow{4}{*}{$\frac{3}{\frac{3}{\pi}}$} & 20 & 1.9 & 2.2 & 12.4 & 0.89 \\
\hline & 40 & 3.1 & 4.6 & 22.1 & 0.77 \\
\hline & 65 & 4.0 & 8.5 & 27.9 & 0.53 \\
\hline & 85 & 4.3 & 9.4 & 33.2 & 0.56 \\
\hline \multirow{4}{*}{$\begin{array}{l}\text { 己े } \\
\text { Фે }\end{array}$} & 20 & 1.9 & 0.7 & 3.9 & 0.89 \\
\hline & 40 & 3.1 & 1.8 & 9.3 & 0.82 \\
\hline & 65 & 4.0 & 3.1 & 14.4 & 0.75 \\
\hline & 85 & 4.3 & 3.9 & 14.7 & 0.60 \\
\hline
\end{tabular}

with nominal $h$. Assuming that the difference between $C_{\text {eff }}^{\prime}$ and $C_{\text {int }}^{\prime}$ is due to extrinsic parasitic capacitances $C_{\text {ext }}^{\prime}$ acting on the same unit of length, we can estimate $C_{\mathrm{ext}}^{\prime}$ (red area in Fig. 4a). The trench depth significantly affects $C_{\text {int }}^{\prime}$, as shown in the simulation in Fig. 4b. For a given $w$, shallower trenches result in larger $C_{\mathrm{int}}^{\prime}$ since the electric field lines from the gate region overlap more of the unetched semiconductor material, whose dielectric constant is larger than that of air in the trenches. Therefore, shallower trenches offer an improved gate control over electrons in the channel, which suggests an effective gate control through the unetched underlying semiconductor material. This supports the results of Fig. 3b, as the better gate control of the Shallow devices resulted in a larger $g_{m}$, and their larger $C_{\text {int }}^{\prime}$ for a given $w$ yielded smaller $\left|V_{\text {th }}\right|$ compared to Deep devices. Other effects may also affect the channel for deeper etchings, such as a larger strain relaxation of the barrier that reduces $n_{s}$ and further shifts $V_{\text {th }}$ towards positive values.

The total effective capacitance estimated as $C_{\text {eff }}=C_{\text {eff }}^{\prime \prime} \times w L$ was in the range of $0.7 \mathrm{aF}$ (20 nm-wide) to $3.9 \mathrm{aF}(85 \mathrm{~nm}$ wide) for the Deep, and $2.2 \mathrm{aF}$ ( $20 \mathrm{~nm}$-wide) to $9.4 \mathrm{aF}$ ( $85 \mathrm{~nm}$ wide) for the Shallow devices (Table I). These are extremely small values of gate capacitance, which are very promising for high frequency applications. Since these devices have not been designed for high frequency measurements, we estimated the cut-off frequencies as $f_{T} \approx g_{m} / 2 \pi C_{\text {eff }}$ up to $0.89 \mathrm{THz}$. Table I summarizes the characteristics of the $50 \mathrm{~nm}$-long IPGFETs.

Figure 5 shows the breakdown voltage measurement for the $165 \mathrm{~nm}$-wide $250 \mathrm{~nm}$-long IPGFET in off-state $\left(V_{g s}=-11 \mathrm{~V}\right)$, revealing a small drain $I_{d s}$ and gate $I_{g}$ leakage currents, below $1 \mathrm{nA}$ and $100 \mathrm{pA}$ respectively, even at $300 \mathrm{~V}$, along with a very

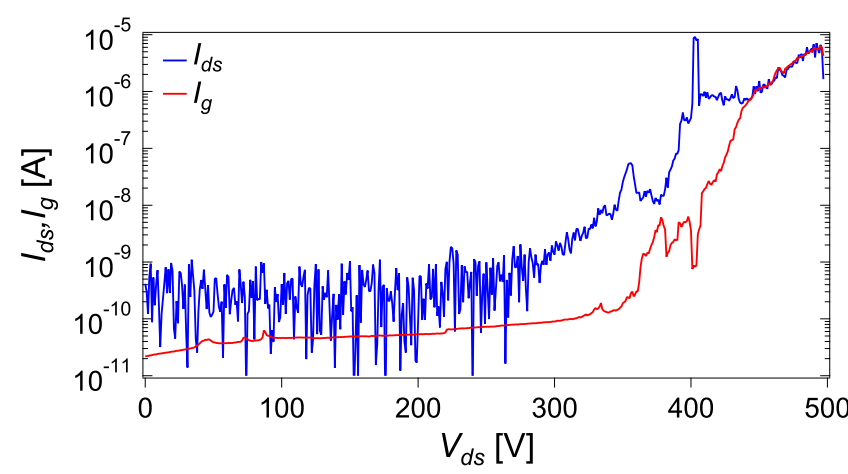

Fig. 5: (a) $I_{d s}$ and $I_{g}$ versus $V_{d s}$ for a $165 \mathrm{~nm}$-wide $250 \mathrm{~nm}$ long IPGFET in off-state $\left(V_{g s}=-11 \mathrm{~V}\right)$.

high breakdown voltage of $500 \mathrm{~V}$ (regardless of the width of the devices). The similar behavior of $I_{d s}$ and $I_{g}$ suggests that at large voltages the leakage current flows entirely through the semiconductor buffer layers, not through the nanowires, since both the drain and gate contacts are ohmic. In addition, the device performance and breakdown characteristics are not hindered by the dielectric quality, since air trenches serve as dielectric in these IPGFETs.

In conclusion, we demonstrated for the first time Al$\mathrm{GaN} / \mathrm{GaN}$ IPGFETs with a large current density of $1.4 \mathrm{~A} / \mathrm{mm}$, $g_{m}$ up to $665 \mathrm{mS} / \mathrm{mm}$ and very large breakdown voltage of $500 \mathrm{~V}$. The in-plane gates resulted in ultra-small gate capacitance down to $0.7 \mathrm{aF}$, leading to an estimated $f_{T}$ up to $0.89 \mathrm{THz}$. These results reveal the enormous potential of III-Nitride IPGFETs for high-frequency and high-power, once this technology is successfully scaled up. Furthermore, the inplane-gate structure could offer an interesting geometry for studying carrier transport in nanoscale channels.

\section{ACKNOWLEDGMENT}

The authors would like to thank the staff in CMi and ICMP cleanrooms at EPFL for technical support.

\section{REFERENCES}

[1] A. D. Wieck and K. Ploog. Inplanegated quantum wire transistor fabricated with directly written focused ion beams. Applied Physics Letters, 56(10):928-930, March 1990. Doi: 10.1063/1.102628.

[2] D. K. de Vries, P. Stelmaszyk, and A. D. Wieck. Intrinsic and extrinsic capacitances of in-plane-gated transistors. Journal of Applied Physics, 79(10):8087, 1996. Doi: 10.1063/1.362364.

[3] A. D. Wieck and K. Ploog. High transconductance inplanegated transistors. Applied Physics Letters, 61(9): 1048-1050, August 1992. Doi: 10.1063/1.107712.

[4] J. Nieder, A. D. Wieck, P. Grambow, H. Lage, D. Heitmann, K. v Klitzing, and K. Ploog. One dimensional lateral field effect transistor with trench gate channel insulation. Applied Physics Letters, 57(25):2695-2697, December 1990. Doi: 10.1063/1.103803.

[5] J. S. McLean, A. D. Wieck, M. Bleder, and K. Ploog. High frequency characteristics of in-plane-gate transistors. Applied Physics Letters, 61(11):1324, 1992. Doi: 10.1063/1.107580. 
[6] K. Ogawa, J. Allam, N. de B. Baynes, J. R. A. Cleaver, T. Mishima, and I. Ohbu. Ultrafast characterization of an inplane gate transistor integrated with photoconductive switches. Applied Physics Letters, 66(10):1228-1230, March 1995. Doi: 10.1063/1.113245.

[7] K. Ogawa, J. Allam, N. De B. Baynes, J. R. A. Cleaver, T. Mishima, and I. Ohbu. Ultrafast characterization of inplane-gate field-effect transistors: parasitics in laterally gated transistors. Optical and quantum electronics, 28 (7):907-917, 1996.

[8] M. Dilger, R. J. Haug, K. Eberl, A. Kurtenbach, Y. Kershaw, and K. v. Klitzing. Transport characterization of inplane gate devices fabricated by direct epitaxial growth on patterned substrates. Applied Physics Letters, 68(22): 3132-3134, May 1996. Doi: 10.1063/1.115801.

[9] U. Meiners, H. Brugger, B. E. Maile, C. Wlk, and F. Koch. Quasi one-dimensional in-plane-gate fieldeffect-transistor. Solid-State Electronics, 37(4):10011004, April 1994. Doi: 10.1016/0038-1101(94)90345-X.

[10] Jan-Olof J. Wesstrm, Katharina Hieke, Bjrn Stlnacke, Thomas Palm, and Bjrn Stoltz. Demonstration of quantized conductance in deeply reactive ion etched In0.53ga0.47as/InP electron waveguides with in-plane gates. Applied Physics Letters, 70(10):1302-1304, March 1997. Doi: 10.1063/1.118518.

[11] Yuji Komatsuzaki, Kazuhiro Higashi, Tomoteru Kyougoku, Koji Onomitsu, and Yoshiji Horikoshi. Negative Differential Resistance in InGaAs/InAlAs Nanoscale In-Plane Structures. Japanese Journal of Applied Physics, 49(10):104001, October 2010. Doi: 10.1143/JJAP.49.104001.

[12] A. Ebbers, D. Reuter, M. Heuken, and A.D. Wieck. In-plane gate transistors in AlxGa1xN/GaN heterostructures written by focused ion beams. Superlattices and Microstructures, 33(5-6):381-388, May 2003. Doi: 10.1016/j.spmi.2004.02.012.

[13] Lars Hiller, Katja Tonisch, and Jrg Pezoldt. Side gate AlGaN/GaN FET on silicon and sapphire. physica status solidi (c), 11(2):280-283, February 2014. Doi: 10.1002/pssc.201300298.

[14] O. Landr, D. Camacho, C. Bougerol, Y. M. Niquet, V. Favre-Nicolin, G. Renaud, H. Renevier, and B. Daudin. Elastic strain relaxation in GaN/AIN nanowire superlattice. Physical Review B, 81(15), April 2010. Doi: 10.1103/PhysRevB.81.153306.

[15] M. Azize and T. Palacios. Top-down fabrication of AlGaN/GaN nanoribbons. Applied Physics Letters, 98 (4):042103, 2011. Doi: 10.1063/1.3544048.

[16] Elison Matioli and Toms Palacios. Room-Temperature Ballistic Transport in III-Nitride Heterostructures. Nano Letters, 15(2):1070-1075, February 2015. Doi: $10.1021 / \mathrm{nl} 504029 \mathrm{r}$.

[17] Giovanni Santoruvo, Adrien Allain, Dmitry Ovchinnikov, and Elison Matioli. Magneto-ballistic transport in $\mathrm{GaN}$ nanowires. Applied Physics Letters, 109(10):103102, September 2016. Doi: 10.1063/1.4962332. 